\title{
Handling Irreducible Loops: Optimized Node Splitting vs. DJ-Graphs
}

\author{
S. Unger, F. Mueller
}

This article was submitted to

Euro-Par 2001, Manchester, UK, August 28-31, 2001

\section{May 10, 2001}

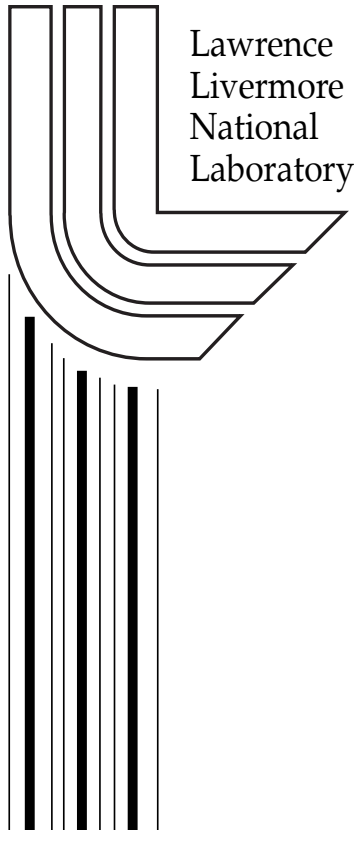




\section{DISCLAIMER}

This document was prepared as an account of work sponsored by an agency of the United States Government. Neither the United States Government nor the University of California nor any of their employees, makes any warranty, express or implied, or assumes any legal liability or responsibility for the accuracy, completeness, or usefulness of any information, apparatus, product, or process disclosed, or represents that its use would not infringe privately owned rights. Reference herein to any specific commercial product, process, or service by trade name, trademark, manufacturer, or otherwise, does not necessarily constitute or imply its endorsement, recommendation, or favoring by the United States Government or the University of California. The views and opinions of authors expressed herein do not necessarily state or reflect those of the United States Government or the University of California, and shall not be used for advertising or product endorsement purposes.

This is a preprint of a paper intended for publication in a journal or proceedings. Since changes may be made before publication, this preprint is made available with the understanding that it will not be cited or reproduced without the permission of the author.

This report has been reproduced directly from the best available copy.

Available electronically at http://www.doc.gov/bridge

Available for a processing fee to U.S. Department of Energy

And its contractors in paper from

U.S. Department of Energy

Office of Scientific and Technical Information

P.O. Box 62

Oak Ridge, TN 37831-0062

Telephone: (865) 576-8401

Facsimile: (865) 576-5728

E-mail: reports@adonis.osti.gov

Available for the sale to the public from

U.S. Department of Commerce

National Technical Information Service

5285 Port Royal Road

Springfield, VA 22161

Telephone: (800) 553-6847

Facsimile: (703) 605-6900

E-mail: orders@ntis.fedworld.gov

Online ordering: http://www.ntis.gov/ordering.htm

\section{OR}

Lawrence Livermore National Laboratory

Technical Information Department's Digital Library

http://www.llnl.gov/tid/Library.html 


\title{
Handling Irreducible Loops: Optimized Node Splitting vs. DJ-Graphs *
}

\author{
Sebastian Unger ${ }^{1}$ and Frank Mueller ${ }^{2}$ \\ 1 DResearch Digital Media Systems, Otto-Schmirgal-Str. 3, 10319 Berlin (Germany) \\ 2 CASC, Lawrence Livermore Nat'l Lab, Box 808, L-561, Livermore, CA 94551, USA \\ frank.mueller@llnl.gov, phone: +1.925.424.3642, fax: +1.925.423.2993
}

\begin{abstract}
This paper addresses the question of how to handle irreducible regions during optimization, which has become even more relevant for contemporary processors since recent VLIW-like architectures highly rely on instruction scheduling. The contributions of this paper are twofold. First, a method of optimized node splitting to transform irreducible regions of control flow into reducible regions is derived. This method is superior to approaches previously published since it reduces the number of replicated nodes by comparison. Second, three methods that handle regions of irreducible control flow are evaluated with respect to their impact on compiler optimizations: traditional and optimized node splitting as well as loop analysis through DJ graphs. Measurements show improvements of $1-40 \%$ for these methods of handling irreducible loop over the unoptimized case.
\end{abstract}

\section{Introduction}

Compilers heavily rely on recognizing loops for optimizations. Most loop optimizations have only been formulated for natural loops with a single entry point (header), the sink of the backedge(s) of such a loop. Multiple entry loops cause irreducible regions of control flow, typically not recognized as loops by traditional algorithms. These regions may result from goto statements or from optimizations that modify the control flow. As a result, loop transformations and optimizations to exploit instruction-level parallelism cannot be applied to such regions so that opportunities for code improvements may be missed.

Modern architectures, such as very long instruction word (VLIW) architectures (Phillips TriMedia, IA-64), require aggressive instruction scheduling to exploit their performance [6] but this requires knowledge about the structure of a program, which contemporary compilers generally do not support for irreducible regions of code. In addition, aggressive global instruction scheduling, enhanced modulo scheduling [13], trace scheduling and profile-guided code positioning combined with code replication $[8,9]$ or applied during binary translation may result in branch reordering and code replication, which itself may introduce irreducible regions. This paper briefly discusses traditional loop splitting, contributes a new approach of optimized node splitting and reports on a performance study of these approaches with DJ-graphs that recognize irreducible loops.

\footnotetext{
* Part of this work was performed under the auspices of the U.S. Department of Energy by University of California Lawrence Livermore National Laboratory under contract No. W-7405-Eng-48.
} 


\section{Traditional Node Splitting}

Node splitting is based on T1/T2-interval analysis that detects irreducible regions in a flow graph. T1/T2 are iteratively applied on the flow graph reducing it to a simpler one:

T1 Remove any edge that connects a node to itself.

T2 A node with only one predecessor are merged into a single abstract node while preserving incoming edges of the predecessor and outgoing edges of the original node.

If these transformations are applied as long as possible the resulting graph is called the limit graph. If the final graph is trivial (a singleton node), then the original flow graph was reducible. Otherwise, all of its nodes either have none or more than one predecessor. Node splitting defines a transformation T3, which is applied on the limit graph:

T3 Duplicate a node with multiple predecessors (one copy per predecessor). Connect each predecessor to a distinct copy and duplicate outgoing edges of the original node.

After the application of T3, apply T1/T2 again and repeat this process. The resulting limit graph is always trivial. If the above process is reversed, leaving the duplicated nodes in place, the result is a reducible flow graph that is equivalent to the original one. This algorithm is inefficient because it does not consider which nodes form the irreducible loops. In this work, algorithms will be presented that exactly analyze the extent, structure and nesting of irreducible loops. Based on such an analysis a much better algorithm than that above will be constructed.

\section{Properties of Irreducible Regions of Code}

The motivation of this work is to develop an algorithm that converts an arbitrary irreducible control flow graph into an equivalent reducible one with the minimal possible growth in code size. This first involves the construction of an algorithm. This work builds on Janssen and Corporaal [7] who found that each irreducible loop has exactly one maximal subset of at least two of its nodes that have the same immediate dominator, which in turn is not part of the loop. They also discovered that these sets play an important role when minimizing the number of splits. Their definition of so-called Shared External Dominator sets was:

Definition 1 (Loop-set). A loop in a flow graph is a path $\left(n_{1}, \ldots, n_{k}\right)$ where $n_{1}$ is an immediate successor of $n_{k}$. The nodes $n_{i}$ do not have to be unique. The set of nodes contained in the loop is called a loop-set.

Definition 2 (SED-set). A Shared External Dominator set (SED-set) is a subset of a loop-set $L$ whose elements share the same immediate dominator and whose immediate dominator (idom) is not part of $L$. A SED-set of $L$ is defined as: $\operatorname{SED}$-set $(L)=\left\{n_{i} \in L \mid \operatorname{idom}\left(n_{i}\right)=e \notin L\right\}$.

Definition 3 (MSED-set). A Maximal Shared External Dominator set (MSED-set) $K$ of a loop-set $L$ is defined as:

SED-set $K$ is maximal $\Longleftrightarrow \nexists$ SED-set $M$, such that $K \subset M$ and $K, M \subseteq L$. The MSED-set is a generalization of the single entry block in natural loops, 


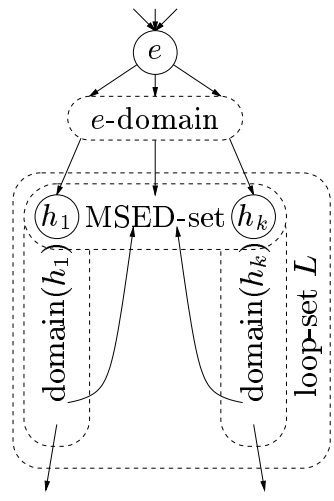

(a) Irred Loop Struct

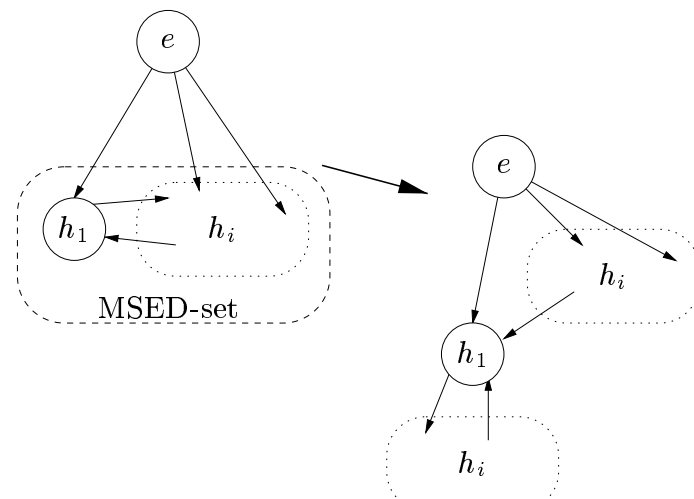

(b) One Step of the Recursive Algorithm $\mathrm{T}_{r}$

Fig. 1. Analyzing Irreducible Loop Structures and Optimized Node Splitting which consists of just one node. In the following, the nodes of MSED-sets will be simply called the header nodes or headers. Building on that, new generalized definitions can also be found for the bodies (an irreducible loop can have more than one), backedges and the nesting of irreducible loops. Figure 1(a) illustrates this generalized structure. Domains represent the body of a natural loop. Edges from a domain back into the MSED-sets are backedges. The node $e$ is the immediate dominator of the header nodes. The region called $e$-domain will be defined and used in the next section.

The following, generalized definitions of backedges and domains are based on MSED-sets whose definition in turn depends on the loop-set. This means that the extension of the loop-set cannot be defined using backedges as it is for natural loops. This is only a problem because the definition of MSED-sets does in no way require the loop-set to be maximal. However, several of the following theorems only hold if the loop-sets are SED-maximal.

Definition 4 (SED-maximal loop-sets). A loop-set $L$ is SED-maximal if there is no other loop-set $L^{\prime}$ such that $L \subset L^{\prime}$ and $M S E D$-set $(L) \subseteq$ $M S E D-\operatorname{set}\left(L^{\prime}\right)$.

Definition 5 (Domains). Let $L$ be an irreducible SED-maximal loop-set, $K$ be its MSED-set and $h_{i}$ be the nodes of $K$. The domain of $h_{i}$ is then defined as: $\operatorname{domain}\left(h_{i}\right)=\left\{n_{j} \in L \mid h_{i}\right.$ dominates $\left.n_{j}\right\}$

Definition 6 (backedges). Let $L$ be an irreducible SED-maximal loop-set, $K$ be its MSED-set and $h_{i}$ be the nodes of $K$. An edge $(m, n)$ with $m \in L$ and $n \in K$ is then called a back-edge of $L$.

Theorem 1. The nodes of $L$ are in $K$ or in exactly one of its domains.

Theorem 2. All edges into domain $(h) \backslash\{h\}$ originate from $h$.

Theorem 3. Let $L_{1}$ and $L_{2}$ be two different, SED-maximal loop-sets, $K_{1}, K_{2}$ their respective $M S E D$-sets and $e_{1}, e_{2}$ the external dominators. Then

- If neither $L_{1} \subset L_{2}$ nor $L_{2} \subset L_{1}$ then $L_{1} \cap L_{2}=\emptyset . \quad$ (distinct loops)

- If $L_{2} \subset L_{1}$ then there is a node $h \in K_{1}$ such that $L_{2} \subset$ domain $(h)$.

(nested loops)

The proofs of these theorems can be found in [12]. The results are used in the following section to develop an optimized algorithm for node splitting. 


\section{Optimized Node Splitting}

The knowledge about the structure of irreducible loops can be used to guide the $\mathrm{T} 3$ transformation to some extend. Repeated application of T1/T2 will collapse domains into their headers leaving an MSED-set. Applying T3 to a node in the MSED-set then splits a header and its entire domain.

As the domains are collapsed into one abstract node, multiple edges from one domain to a single header node will reduce to just one edge from the abstract node to the header node. This reduces the number of copies of that node and is also true for multiple edges from the outside. Figure 1(a) suggests by the naming that the region called $e$-domain (defined below) should be handled just as any other domain. That is, transformations $\mathrm{T} 1$ and $\mathrm{T} 2$ should collapse it into $e$, thereby reducing multiple edges from that domain to any header node into one edge. Of course, T3 should not be applied to this abstract node.

Definition 7 (e-domain). Let $L$ be an irreducible SED-maximal loop-set, $K$ be its MSED-set and e the external dominator. That is: If $e$ is the immediate dominator of the nodes in $K$, then the set e-domain is defined as:

$$
e \text {-domain }=\left\{\begin{array}{l|l}
n_{i} \in N & \begin{array}{l}
\text { e dominates } n_{i}, n_{i} \notin L \text { and } \\
\exists \text { a path } p \text { from } n_{i} \text { into } L \text { with } \\
e \notin p
\end{array}
\end{array}\right\}
$$

Does this algorithm always produce the minimal reducible equivalent flow graph? Unfortunately not, as counter-examples show that selecting the nodes to split just by their weight is not sufficient. Another question is if there is always an order that leads to the minimum. Alas, not even that is true. Figure 2 gives a flow graph where no order will split the nodes to yield a minimal graph.

A new approach has been developed, based on the observation that all of the counter-examples contained one of the header nodes that was not split at all. The new approach chooses a single header node (plus its domain, of course) that not split at all. All other nodes of the loop-set are split once. This is illustrated in Figure 1(b). The regions containing the copies of the remaining nodes of $L$ are not yet guaranteed to be reducible but they are guaranteed to be smaller than $L$ by at least one node. Hence, the above step can be applied recursively to these copied regions. This new approach also needs a scheme for selecting the node $h_{1}$ with the advantage over the previous approach that for any flow graph there is a selection scheme that leads to a minimal result. All that remains is to

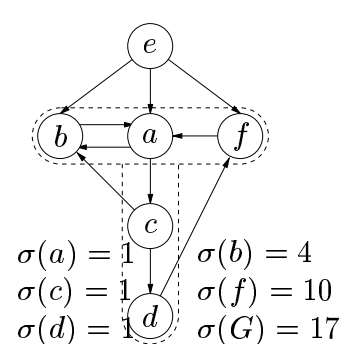

(a) Orig. $\mathrm{CFG}$

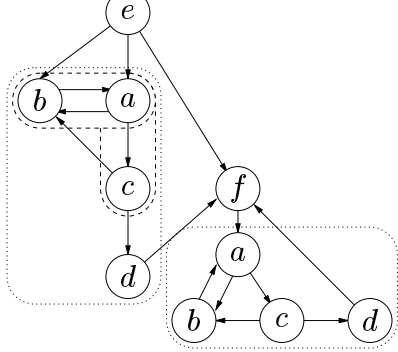

(b) freeze $f$ and apply $\mathrm{T}_{r}$

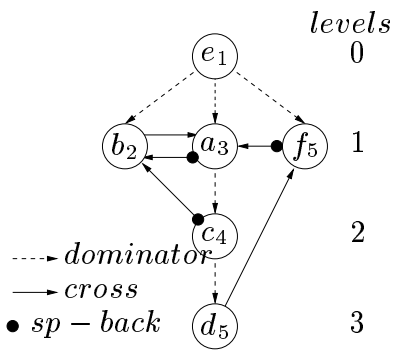

(c) DJ-Graph for 2(a)

Fig. 2. T3 Cannot Split this Graph with Weights $\sigma$ in a Minimal Way. 
actually find this scheme. First, however, the algorithm is defined more precisely. The following notation will be used in the following: If $f$ is a function over the nodes of any control flow graph, then $f(X)$, where $X$ is a subset of these nodes, stands for the set $\{f(x) \mid x \in X\}$.

Definition 8 (Transformation $\mathbf{T}_{r}$ ). Let $G=(N, E, s)$ be an arbitrary (irreducible) control flow graph, $L$ an $S E D$-maximal, irreducible loop-set of $G, K$ its MSED-set, e the external dominator and $h$ an arbitrary node from $K$. Then the transformation $G^{\prime}=\left(N^{\prime}, E^{\prime}, s^{\prime}\right)=T_{r}(G, L, h)$ is defined as follows (with $S=(L \backslash$ domain $(h)))$ :

$-N^{\prime}=(N \times\{1\}) \cup(S \times\{2\})$

$-E^{\prime} \subset N^{\prime} \times N^{\prime}$ such that the following restrictions hold:

- $(x, y) \in E \wedge(x, y) \notin(\operatorname{domain}(h) \times S) \Longleftrightarrow((x, 1),(y, 1)) \in E^{\prime}$

- $(x, y) \in E \wedge(x, y) \in(\operatorname{domain}(h) \times S) \Longleftrightarrow((x, 1),(y, 2)) \in E^{\prime}$

- $(x, y) \in E \wedge(x, y) \in(S \times(N \backslash S)) \Longleftrightarrow((x, 2),(y, 1)) \in E^{\prime}$

- $(x, y) \in E \wedge(x, y) \in(S \times S) \Longleftrightarrow((x, 2),(y, 2)) \in E^{\prime}$

$-s^{\prime}=(s, 1)$

The above transformation represents one step of the algorithm. All nodes of the loop-set $L$, except for those in the selected header node's domain, are split. The new copies of these nodes are represented by the syntactical construction $S \times\{2\}$ while the old nodes are represented by $N \times\{1\}$. In other words, a single $\mathrm{T}_{r}$ transformation results in one split to copy $S$ so that unnecessary copies are avoided. For example, applying $\mathrm{T}_{r}$ once on the graph of Fig. 2(a) without copying node $f$ results in Fig. 2(b). A proof of the correctness of optimized node splitting using $\mathrm{T}_{r}$ is beyond the scope of this paper but can be found elsewhere [12]. The recursive algorithm for $\mathrm{T}_{r}$ is shown in the appendix.

\section{Using DJ Graphs to Optimize Irreducible Loops}

The representation of DJ Graphs [11] may be used for incremental data-flow analysis but it also provides the means to perform loop optimizations on irreducible loops. By constructing the DJ Graph of a control-flow graph, natural and irreducible loops and their nesting hierarchy can be detected.

An example is depicted in Figure 2(c). The DJ-Graph consists of the edges of the dominator tree (dashed), backedges, and the remaining edges of the control flow called cross edges (solid). Furthermore, sp-back edges are control-flow edges $x \rightarrow y$ where $x=y$ or $y$ is an ancestor of $x$ is a spanning tree resulting from a depth-first search. In the example, a search in the order of the indicies of the nodes indicates that the edges marked with bullets are sp-back. Loops in the DJ-Graph can then be found starting from the lowest dominator level (level 3). If a backedge exists at the current level, then nodes corresponding to its natural loop are collapsed into one node. Afterwards, if a cross edge is also sp-back, all strongly connected components at the current level or below represent an irreducible loop and are collapsed to a single node before considering the next higher level. In the example, there are no backedges but several cross edges at level 1 that are also sp-back. The only strongly connected component comprises all nodes at level 1 or below, i.e., exactly one irreducible loop is found. However, Figure 2(b)shows that an inner loop $\{a, b\}$ and an outer loop $\{a, b, c\}$ may be 
distinguished by optimized node splitting. Nonetheless, DJ-Graphs still allow the distinction of irreducible loop bodies either if they comprise different levels or if they represent distinct strongly connected components. Furthermore, DJ-Graphs also allow the detection of reducible loops within irreducible ones. Had there been an edge $d \rightarrow c$ in Figure 2(c), then this edge would have been recognized as a backedge whose source and sink comprise a loop at level 2 .

There are other differences between natural loops and DJ-graphs representations of irreducible loops. Instead of one loop header for natural loops, irreducible loops have multiple entry blocks with predecessor blocks outside the loop. Furthermore, there is no block in an irreducible loop that dominates all other blocks within the loop. Notice, however, that we allow a natural loop to share a header with an irreducible loop. We still distinguish both loops in this case. These differences require changes to other loop optimizations.

Code motion moves invariant operations out of the body of a natural loop into the preheader block. For irreducible loops, the set of entry blocks can be augmented by a set of preheader blocks. Then, a copy of a loop-invariant operation is moved into all preheaders at once. Code motion as stated in [1] applies with minor changes, e.g., to find invariant statements in loop $l$ :

1. $d s t=s r c$ is invariant if $s r c$ is constant or its reaching definitions are outside $l$, as indicated by registers live on entry for each preheader of $l$.

2. transitively mark statements in step 3 until no more unmarked invariant statements are found.

3. $d s t=s r c$ is invariant if $s r c$ is constant, if its sole reaching definition inside

$l$ is marked invariant or if its reaching definitions are outside $l$.

For each entry of an irreducible loop, we delete all other entries and collect the sources of all backedges within the resulting region. Notice that such a region may contain more than one natural loop now. We call the collected blocks the sources of pseudo-backedges of the irreducible loop. A block of an irreducible loop is executed during each iteration if it dominates all sources of pseudo-backedges within the corresponding reducible regions. This requires dominator information of the reducible pseudo-regions to be associated with an irreducible loop.

Finding induction variables becomes more complicated due to irreducible loops. We limit our approach by requiring that changes to induction variables are performed in blocks which are executed on each loop iteration. This information is already available from code motion for memory reads. In addition, one could allow balancing modifications in corresponding conditional arms. These arms range from a split at an always iterated block to a join at the next block that is always executed during each loop iteration. We did not implement this extension. Once induction variables are identified, strength reduction and induction variable elimination are performed as for natural loops, except that invariant operations of register loads are moved into all preheaders of the irreducible loop.

Similar to the handling of induction variables, recurrences can be optimized by moving the prologue into all preheaders, given that the memory access originates in a block that is executed on each loop iteration. Other optimizations also benefit from the additional loop information. For example, global register allocation is performed by prioritized graph coloring in VPO. The priority is based on 
the loop frequency, which is readily available even for irreducible loops and their nesting within other loops. No modification was required to such optimizations.

\section{Measurements}

We chose VPO [3] as a platform to conduct a performance evaluation. VPO only recognizes natural loops with a single header, just as all contemporary optimizing compilers we know of. Irreducible regions of code remain unoptimized. First, we added the recognition of DJ Graphs to VPO, extended code motion, strength reduction, induction variable elimination and recurrences. Second, we implemented optimized node splitting through $\mathrm{T}_{r}$. The heuristic driving node selection was to choose the header of the domain with the most instructions. This node (and its domain) were not split while all other nodes in the loop set were split. Third, traditional node splitting using T1/T2/T3 was integrated. The heuristic considers for each header the number of instructions times predecessors. The header with the smallest heuristic value is then chosen.

Test programs with irreducible loops were used to measure the effect of the three different approaches. Dfa simulates a deterministic finite automata representing an irreducible loop containing two independent natural loops (see Fig. $2 \mathrm{a}$ in [11]). Arraymerge, extracted and translated from a Fortan application, merges two sorted arrays. The remaining programs are common UNIX utilities.

The measurements were collected for the Sun SPARC architecture using the environment for architectural study and experimentation (EASE) that is integrated into VPO. The left part of Table 1 depicts the number of dynamically executed instructions of a function that was originally irreducible. Changes in percent are reported relative to not optimizing irreducible loops. The table shows comparable reductions of $1-40 \%$ in the number of executed instructions for DJ, $\mathrm{T} 3$ and /TR. The improvements for $\mathrm{T}_{r}$ can be attributes in part to VPO, which still applies loop optimizations to reducible loops contained in the same function as irreducible ones. Other compilers may suppress optimizations resulting in even higher gains for DJ or $\mathrm{T}_{r}$. The quantity of improvements are subject to the execution frequency of irreducible regions within the enclosing function. For

\begin{tabular}{|c|c|c|c|c|c|c|}
\hline \multirow{2}{*}{ Program } & \multirow{2}{*}{$\begin{array}{c}\text { DJ } \\
\text { Graph }\end{array}$} & \multicolumn{2}{|c|}{ Node Splitting } & \multirow{2}{*}{$\begin{array}{c}\text { DJ } \\
\text { Graph }\end{array}$} & \multicolumn{2}{|c|}{ Node Splitting } \\
\hline & & T3(trad.) & $\mathrm{T}_{r}$ (opt.) & & T3(trad.) & $\mathrm{T}_{r}$ (opt.) \\
\hline dfa & $-13.91 \%$ & $-13.85 \%$ & $-13.88 \%$ & $-4.79 \%$ & $+30.54 \%$ & $+21.56 \%$ \\
\hline arraymerge & $-36.76 \%$ & $-39.70 \%$ & $-39.70 \%$ & $-0.83 \%$ & $+32.50 \%$ & $+19.17 \%$ \\
\hline tail & $0.00 \%$ & $0.00 \%$ & $0.00 \%$ & $+1.70 \%$ & $+6.60 \%$ & $+3.83 \%$ \\
\hline unifdef1 & $-0.36 \%$ & $+9.01 \%$ & $+10.37 \%$ & $+7.14 \%$ & $+26.79 \%$ & $+28.57 \%$ \\
\hline unifdef2 & $-4.40 \%$ & $-7.23 \%$ & $-1.10 \%$ & $+1.79 \%$ & $+21.43 \%$ & $+25.00 \%$ \\
\hline hyphen & $+0.01 \%$ & $+0.01 \%$ & $+0.01 \%$ & $+8.93 \%$ & $+20.24 \%$ & $+20.24 \%$ \\
\hline cpp & $+0.05 \%$ & $-1.61 \%$ & $-1.18 \%$ & $-0.27 \%$ & $+1.64 \%$ & $+2.33 \%$ \\
\hline nroff1 & $-8.28 \%$ & $-7.19 \%$ & $-13.50 \%$ & $+0.84 \%$ & $+35.15 \%$ & $+10.46 \%$ \\
\hline nroff2 & $-4.37 \%$ & $-4.42 \%$ & $-0.19 \%$ & $+0.47 \%$ & $+25.58 \%$ & $+1.86 \%$ \\
\hline nroff3 & $0.00 \%$ & $+0.06 \%$ & $+0.13 \%$ & $0.00 \%$ & $+16.67 \%$ & $+9.80 \%$ \\
\hline sed & $-0.59 \%$ & $-4.02 \%$ & $-4.49 \%$ & $+2.06 \%$ & $-0.40 \%$ & $-1.27 \%$ \\
\hline & \multicolumn{3}{|c|}{ Dynamically Executed Instr. } & \multicolumn{3}{|c|}{ Static Code Size (Instr.) } \\
\hline
\end{tabular}

Table 1. Instructions and their Changes for Irreducible Regions 
example, Arraymerge contains a central loop for sorting that was irreducible. Tail, on the other hand, contains an irreducible loop for block reads, which is executed infrequently relative the the other instructions within the function. The function "skipcomment" in Unifdef1 showed worse results for $\mathrm{T}_{r}$, which is correlated to fewer delay slots of branches being filled. Similar effects were observed for cases where little changes were observed.

The right part of Table 1 depicts for a function of a program containing an irreducible loop the size of the function in number of instructions. The code size only changes insignificantly for DJ-Graphs. These small changes are due to other optimizations. The quantity of changes depends on the number of preheaders and the compensation by other optimizations, such as peephole optimization. For $\mathrm{T}_{r}$, the code size changes between $-1 \%$ and $28 \%$. This change in size is measured relative to the original function containing an irreducible loop. The change in code size relative to the entire program was between $0.5 \%$ and $3.5 \%$ for larger test programs and $8-17 \%$ where the irreducible loop comprised most of the test program (Dfa, Arraymerge and Hyphen). The fact that node splitting stops at function boundaries limits the overall increase in code size for the entire program so that exponential growth was not encountered in the experiments and is unlikely in general. T3 resulted in more code growth (up to 35\%). T3 mostly shows a differnt dynamic instruction count than $\mathrm{T}_{r}$ indicating that $\mathrm{T}_{r}$ reduces the amount of code duplication while preserving the performance.

The differences between the two node splitting techniques are further illustrated in Table 2 depicting the number of copied register transfer lists (RTLs) for $\mathrm{T} 3$ and $\mathrm{T}_{r}$ (changes relative to $\mathrm{T} 3$ parenthesis). Since both node splitting approaches are performed as one of the first optimizations, each RTL of the intermediate code representation resembles a very simplistic instruction. The numbers show that the traditional method results in significantly more replicated code after node splitting than the $\mathrm{T}_{r}$, which only requires $1-30 \%$ of copied RTLs under $\mathrm{T}_{r}$ relative to $\mathrm{T} 3$. T3 may yield considerably inferior results that $\mathrm{T}_{r}$ for optimizing compilers with less aggressive optimizations than VPO. These findings indicate that $\mathrm{T}_{r}$ is superior to the traditional approach but actual savings depend on the phase ordering of optimizations and the infrastructure of the optimizing compiler as such.

In addition, we compared the node splitting methods T3

\begin{tabular}{|l|r|r|}
\hline Program(Function) & \multicolumn{1}{|c|}{ T3 } & \multicolumn{1}{|c|}{$\mathrm{T}_{r}$ (opt.) } \\
\hline dfa(main) & 701 & $204(-70.90 \%)$ \\
arraymerge(MergeArrays) & 306 & $50(-83.66 \%)$ \\
tail(main) & 1906 & $100(-94.75 \%)$ \\
unifdef1(skipcomment) & 218 & $56(-74.31 \%)$ \\
unifdef2(skipquote) & 191 & $44(-76.96 \%)$ \\
hyphen(main) & 914 & $153(-83.26 \%)$ \\
cpp(cotoken) & 2791 & $71(-97.46 \%)$ \\
nroff1(text) & 975 & $138(-85.85 \%)$ \\
nroff2(getword) & 787 & $103(-86.91 \%)$ \\
nroff3(suffix) & 417 & $28(-93.29 \%)$ \\
sed(fcomp) & 4897 & $38(-99.22 \%)$ \\
\hline
\end{tabular}

Table 2. RTLs copied during Node Splitting and $\mathrm{T}_{r}$ with the controlled node splitting (CNS) with heuristic by Janssen and Corporaal [7]. The CNS approach is detailed in the related work section. The measurements indicated that CNS differed only insignificantly from our T3 ap- 
proach, both in the number of executed instructions and the change in code size. Careful analysis revealed that the heuristic used for T3 almost always picked the same nodes for splitting as CNS. Further restrictions on node selection by CNS only occurred in one case (nroff) but had hardly any effect on the results.

Finally, we also measured the instruction cache performance for a $4 \mathrm{kB}$ and 512B direct-mapped cache using VPO and EASE. The hit ratio did not change significantly (less than 1\%) for the tested programs, regardless of the cache size. For changing code sizes, the cache work is often a more appropriate measurement [8], where a miss accounts for 10 cycles delay (for going to the next memory level) and a hit for one cycle. The methods of handling irreducible loops all resulted in reduced cache work for most cases, varying between a reduction of $6 \%$ and $28 \%$. This reduction seems to indicate that execution in replicated regions tends to be localized, i.e., once such a region is entered, executing progresses within this replica rather than transferring control between different replicas.

\section{Related Work}

Reducible flow graphs were first mentioned by Allen [2]. The idea of node splitting stems from Cocke and Miller [4]. DJ Graphs are due to Sreedhar et. al. [11]. Havlak proposed a method for recognizing reducible and irreducible loops as well as the nesting of either ones [5]. His algorithm used node splitting only for headers of natural loops contained within irreducible loops as a means to have distinct header nodes. This work did not use node splitting to make irreducible loops reducible, whereas our work did. Furthermore, our notion of backedges is independent of any graph traversals while Havlak's backedges for irreducible loops depend on the order of a traversal of the control flow. Ramalingam [10] contributed performance improvements and a common formal framework for three schemes for recognizing loop structures, including those by Sreedhar et al. and Havlak. Since our study is concerned with the performance of the compiled programs rather than the performance of the compiler, we did not implement his improvements. However, we strengthen the results of [10] through our structural definition of loops and our SED-maximal loop sets that capture the loop descriptions of previous work and represent a minimal loop nesting forest. In particular, we reduce irreducible loops into reducible ones in a bottom-up fashion (wrt. the level in the dominator tree) by isolating (and freezing) the largest domain and its header while splitting the remaining nodes in the loop set. Recursive splitting ensures that different loops within one irreducible region can be isolated. Hence, we go beyond the approach by Sreedhar et al. although our algorithm uses the same data structures. We also showed how several optimization methods for reducible graphs can be transformed into methods for irreducible graphs, which, once again, strengthens Ramalingam's results [10].

The notion of MSED-sets is introduced by Janssen and Corporaal [7], and a node-splitting algorithm, called "Controlled Node Splitting", is presented that tries to minimize the number of splits. However, their algorithm differs from our approach in that they use the traditional approach of splitting one node while we exclude one node from splitting and split all other nodes in the MSED-set. They report reductions in code sizes to almost 1/10 of the original size. Our 
measurements indicated that these savings were mostly due to the heuristic for node selection. We can also show that their formulation seldom leads to savings in practise while ours handles nested irregular regions more elegantly [12].

\section{Conclusion}

We derived a new approach for optimized node splitting that transforms irreducible regions of control flow into reducible ones. This method is superior to approaches previously published since it reduces the number of replicated nodes by comparison. We also discussed the application of DJ Graphs to recognize the structure of irreducible loops and implemented extensions to common code optimizations to handle these new types of loops. Measurements show improvements of $1-40 \%$ in the number of executed instructions for the approaches of handling irreducible loops. Optimized node splitting has the advantage that it does not require changes to other code optimizations within the compiler but may increase the code size of large programs by about $2 \%$ and the size of small programs by about $12 \%$. On the average, it results in less code growth than traditional node splitting and, hence, is superior to it.

\section{References}

1. A. V. Aho, R. Sethi, and J. D. Ullman. Compilers - Principles, Techniques, and Tools. Addison-Wesley, 1986.

2. F. Allen. Control flow analysis. Sigplan Notices, 5(7):1-19, 1970.

3. M. E. Benitez and J. W. Davidson. A portable global optimizer and linker. In ACM SIGPLAN Conf. on Programming Language Design and Impl., pages 329338, June 1988.

4. J. Cocke and J. Miller. Some analysis techniques for optimizing computer programs. In 2nd Hawaii Conference on System Sciences, pages 143-146, 1969.

5. P. Havlak. Nesting if reducible and irreducible loops. ACM Trans. Programming Languages and Systems, 19(4):557-567, July 1997.

6. J. Hoogerbrugge and L. Augusteijn. Instruction scheduling for trimedia. Journal of Instruction-Level Parallelsim, 1(1-2), 1999. www.jilp.org.

7. J. Janssen and H. Corporaal. Making graphs reducible with controlled node splitting. ACM Trans. Programming Languages and Systems, 19(6):1031-1052, November 1997.

8. F. Mueller and D. B. Whalley. Avoiding unconditional jumps by code replication. In ACM SIGPLAN Conf. on Programming Language Design and Impl., pages 322 330, June 1992.

9. F. Mueller and D. B. Whalley. Avoiding conditional branches by code replication. In ACM SIGPLAN Conf. on Programming Language Design and Impl., pages 5666, June 1995.

10. G. Ramalingam. On loop, dominators, and dominance frontier. In ACM SIGPLAN Conf. on Programming Language Design and Impl., pages 233-241, June 2000.

11. V. Sreedhar, G. Gao, and Y. Lee. Identifying loops using DJ graphs. ACM Trans. Programming Languages and Systems, 18(6):649-658, November 1996.

12. S. Unger and F. Mueller. Handling irreducible loops: Optimized node splitting vs. dj-graphs. TR 146, Inst. f. Informatik, Humbolt University Berlin, January 2001. www.informatik.hu-berlin.de/ ${ }^{\sim}$ mueller.

13. Nancy J. Warter, Grant E. Haab, Krishna Subramanian, and John W. Bockhaus. Enhanced modulo scheduling for loops with conditional branches. In 25th Annual International Symposium on Microarchitecture (MICRO-25), pages 170-179, 1992. 